\title{
KOMPASS
}

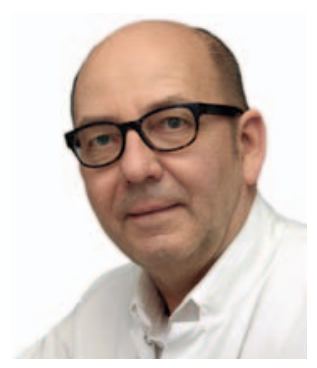

Uwe Pleyer

Klinik für Augenheilkunde

Charité - Universitätsmedizin Berlin

Willkommen zur nunmehr 4. Ausgabe 2017 von Karger Kompass Ophthalmologie! Das Konzept kompakter Wissensvermittlung hat sich inzwischen bei uns Ophthalmologen gut etabliert und erfreut sich zunehmenden Zuspruchs. Bei der Fülle neuer Informationen, die uns täglich erreichen, und vor dem Hintergrund unseres facettenreichen Fachs ist dies allzu gut verständlich.

Dies gilt insbesondere für den Schwerpunkt dieses Heftes: «Uveitis». Für den Augenarzt stellen intraokulare Entzündungen immer wieder eine besondere diagnostische und therapeutische Herausforderung dar. Bereits beim ersten Patientenkontakt stellen sich grundlegende Fragen: Liegt eine infektiöse Genese vor? Sind Systemerkrankungen differentialdiagnostisch zu berücksichtigen? Welche anti-inflammatorischen oder immunmodulatorischen Behandlungsmöglichkeiten sind zu wählen? Unsere differentialdiagnostischen Überlegungen und Möglichkeiten haben sich ständig erweitert, das Therapiespektrum hat sich in den letzten Jahren erfreulich verbreitert und verbessert.

Drei wesentliche Aspekte tragen dazu bei, dass sich dieser Bereich in der Ophthalmologie momentan sehr spannend entwickelt:

1) Enormer Wissenszuwachs. Dies betrifft v.a. die molekulare Biomedizin, die wiederum aus einer Vielzahl anderer Teilbereiche wie z.B. der Immunologie und Genetik gespeist wird. Gleichzeitig ha-
Karger Kompass Ophthalmol 2017;3:152-154 DOI: 10.1159/000481360

\section{Uveitis - (K)ein Enigma}

ben sich die technischen Verfahren, insbesondere die Bildverarbeitung, deutlich verbessert und uns neue Möglichkeiten zur Diagnostik und Verlaufsbeurteilung beschert.

2) Interdisziplinarität. Die Zusammenarbeit mit Kollegen aus anderen Fachbereichen ist nicht neu, wird aber durch viele aktuelle Aspekte noch zwingender notwendig. Dies gilt u.a. für neue hochpotente Therapeutika. Gleichermaßen sind alte und neue Infektionserkrankungen in den Fokus der Abklärung bei intraokularer Entzündung gerückt.

3) Öffentliches Bewusstsein. Das Interesse und Engagement betroffener Patienten, aber auch zunehmender Rückhalt aus Industrie und Politik befördern diesen Bereich der Ophthalmologie. Intraokulare Entzündungen gelten als «orphan disease» und unterliegen damit besonderen Gegebenheiten. Dies betrifft oft eine Solidarisierung der betroffenen $\mathrm{Pa}$ tienten (Stichwort: Selbsthilfegruppen). Gleichzeitig stellen «seltene Erkrankungen» besondere Fördermöglichkeiten in Wissenschaft und Industrie in Aussicht. Es ist erfreulicherweise erkennbar, dass sich gerade die Behandlungsoptionen in den letzten 10 Jahren deutlich verbessert haben.

In dem vorliegenden Themenheft werden zentrale, aktuelle Fragestellungen aufgegriffen. Der Übersichtsbeitrag zum Thema okuläre Toxoplasmose (OT) rückt die häu- figste Infektion der posterioren Uveitis in den Mittelpunkt [1]. Bei ca. 20\% unserer Patientenkohorte (> 10000 Betroffene) an der Charité kann eine intraokulare Infektion als Ätiologie herausgestellt werden. OT gilt als weltweit häufigste Parasitose und weist weiterhin viele offene Fragen auf. Aktuelle Untersuchungen, die mit dem Robert Koch-Institut durchgeführt wurden, zeigen eine weiterhin sehr hohe Seroprävalenz (ca. 60\% der 50-jährigen Normalbevölkerung). Gleichzeitig bleiben die Behandlungsmöglichkeiten dieser Infektion sehr begrenzt. Trifft das Diktum zu, dass die Vielzahl unterschiedlicher Therapien direkt proportional zum Behandlungsversagen einer Erkrankung ist, dann ist die OT ein treffendes Beispiel. Die Liste bisher angewendeter Wirkstoffe ist lang. Fragen der möglicherweise genetisch determinierten Erreger-Wirt-Interaktion bleiben spannend, aber momentan unbeantwortet. Neue Möglichkeiten der Diagnostik und des Monitorings - nicht zuletzt befördert durch neue bildgebende Verfahren - haben das Interesse an dieser alten «Infektionserkrankung» wiederbelebt.

Nach diesem interessanten Auftakt schlieBen sich Berichte über weitere Entwicklungen im Bereich der Ophthalmologie an. Dies betrifft u.a. neue immunmodulatorische Ansätze aus der Neurologie, z.B. zur Behandlung der multiplen Sklerose (MS) mit Fingolimod [2]. Da MS als Autoimmunerkrankung viele Parallelen zur autoimmu-

\section{KARGER}

Fax +4976145207 14 information@karger.com www.karger.com

\section{(c) 2017 S. Karger GmbH, Freiburg}

Accessible online at: www.karger.com/kop
Prof. Dr. Uwe Pleyer, FEBO

Klinik für Augenheilkunde

Charité - Universitätsmedizin Berlin, Campus Virchow-Klinikum

Augustenburger Platz 1, 13353 Berlin, Deutschland

uwe.pleyer@charite.de 
nen Uveitis aufweist, ist es naheliegend, grundlegende Mechanismen zu vermuten und ähnliche Therapieoptionen zu prüfen. Umso überraschender sind zum Teil paradoxe Erscheinungen, wie sie in dem Report von Mack et al. berichtet werden: Bereits in den Zulassungsstudien dieses ersten, oral verfügbaren MS-Präparates (Fingolimod, FTY 720) wurde beobachtet, dass bei höherer Dosierung die Blut-Retina-Schranke beeinflusst wird und ein Makulaödem als Folge einer Blut-Retina-Schrankenstörung auftreten kann. Diese Barrierestörung kann offensichtlich auch im vorderen Augensegment auftreten und wird, wie hier beschrieben, als akute anteriore Uveitis manifest. Da Uveitis und MS bei einem Teil der Patienten assoziiert sind, ist oft eine gemeinsame Therapiestrategie notwendig - ein wichtiger klinischer Aspekt.

Im «Spotlight» stellt Frau Prof. Dr. Nicole Stübiger aktuelle Beiträge zum Thema Uveitis und Rheuma vor [3]. Rheumatische Erkrankun-

gen treten oft mit Augenbeteiligung auf und können Menschen aller Altersklassen betreffen. Auch hier sind eine gründliche Anamnese und eine interdisziplinäre Betreuung des Patienten von hoher Wichtigkeit für Diagnose und Therapie.

Zu dieser erneut sehr interessanten Ausgabe von Karger Kompass Ophthalmologie kann ich den Kollegen des Beirates nur gratulieren. Ihnen als Leser wünsche ich spannende und erkenntnisreiche Lektüre.

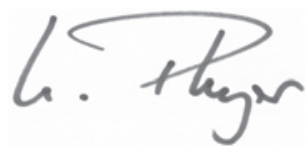

Prof. Dr. Uwe Pleyer

\section{Literatur}

1 Ozgonul C, Besirli CG: Aktuelle Entwicklungen in der Diagnose und Therapie der okulären Toxoplasmose. Karger Kompass Ophthalmol 2017;3:156-166.
2 Mack HG, Tien MC, White OB: Akute Uveitis Anterior bei einem mit Fingolimod (FTY720) behandelten Patienten mit multipler Sklerose. Karger Kompass Ophthalmol 2017;3:197-199.
3 Stübiger N: Beiträge zu intraokularen Entzündungen - Schnittstelle Rheumatologie. Karger Kompass Ophthalmol 2017;3:180-181. 


\section{FOKUS}

\section{Intraokulare Entzündungen}

\section{(10) Epidemiologie}

Es wird geschätzt, dass ca. 400000 Menschen in Deutschland von einer Uveitis betroffen sind und etwa 8000 bis 15000 Neuerkrankungen jährlich auftreten. Die Inzidenz aller Uveitiden beträgt ca. 50 auf 100000 Einwohner pro Jahr (in Mitteleuropa und den USA) [1]. Damit stehen intraokulare Entzündungen zusammen mit der diabetischen Retinopathie in der Altersgruppe der 20- bis 40-Jährigen an erster Stelle für schwerwiegende Sehschädigungen. Eine frühzeitige und adäquate Versorgung vor Manifestation bleibender Schäden ist somit besonders wichtig [2]. Im Gegensatz zu diversen anderen Augenerkrankungen tritt die Uveitis hauptsächlich bei jungen Menschen auf. Etwa 13\% der Betroffenen sind Kinder und Jugendliche. Bezogen auf die Lebenszeit der betroffenen Patienten rückt die Bedeutung intraokularer Entzündungen noch weiter in den Vordergrund. Während die Risiken der diabetischen Retinopathie dem Augenarzt gut bekannt sind, wird die Prognose der chronisch verlaufenden Uveitis oft unterschätzt. In einer Untersuchung zur sozialen und ökonomischen Situation von Uveitispatienten in Großbritannien war im Langzeitverlauf bei 70\% der Augen eine Visusminderung auf >0,3 eingetreten; bei 50\% waren beide Augen betroffen [3]. Für den Augenarzt stellen intraokulare Entzündungen oft eine besondere Herausforderung dar. Es gilt ein breites Spektrum infektiöser Ursachen und Systemerkrankungen differenzialdiagnostisch zu berücksichtigen. Bei ca. 60\% unserer Patienten liegt eine spezifische Genese zugrunde. Dies können Systemerkrankungen (z.B. juvenile rheumatoide Arthritis, Sarkoidose), aber auch Infektionen wie z.B. Viren (Herpes, Rubella), Protozoen (Toxoplasma gondii) und Bakterien (Treponemata, Tuberkulose) sein. Zunehmend müssen auch «Maskerade Syndrome» (intraokulare Lymphome, Karzinom-assoziierte Retinopathie) in Betracht gezogen werden.

\section{Herausforderungen und Handlungsbedarf}

Symptome und Befunde sind sehr vielfältig und können die Diagnose oft verzögern. Da die Beschwerden (z.B. Visusminderung) bei intermediärer oder posteriorer Uveitis oft schleichend eintreten, handelt es sich immer wieder auch um «Zufallsbefunde». Gerade bei Kindern mit juveniler Arthritis liegt überwiegend ein äußerlich «weißes Auge» vor, wodurch die Krankheit oft nur zufällig und verzögert entdeckt wird [4].

Irreversible Schäden können meist durch eine frühzeitige Behandlung vermieden werden. Dies weist auf die Bedeutung der interdisziplinären Zusammenarbeit mit Fachärzten aus anderen Disziplinen hin. Sie sind nicht nur für die diagnostische Abklärung, sondern auch in der Betreuung und Behandlung mit (systemischen) immunmodulierenden Wirkstoffen wichtig [5].

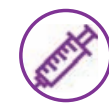

Therapieoptionen

Die Therapiemöglichkeiten sind erfreulicherweise in den letzten Jahren deutlich vielfältiger geworden. Dies betrifft insbesondere die Behandlung der problematischen Entzündungen des hinteren Augensegmentes (intermediäre, posteriore und Panuveitis). Sie nehmen ca. 40\% bis 50\% aller nicht infektiösen, intraokularen Entzündungen ein. Die Anwendung von Biologika und «Small Molecules» hat in vielen anderen Bereichen der Medizin bereits länger Einzug gehalten. Inzwischen beginnen sie sich auch bei der Behandlung der autoimmun vermittelten Uveitis zu etablieren. Die systemische Therapie mit TNF-Blockern, Interferon und Interleukin-6-Antagonisten sowie die intravitreale Injektion verschiedener Wirkstoffe (Dexamethason, Sirolimus) sind hier zu hervorzuheben [5-7].

\section{Literatur}

1 Berufsverband der Augenärzte Deutschlands e.V., Deutsche Ophthalmologische Gesellschaft e.V.: Leitlinie Nr. 14: Uveitis anterior. www.dog.org/wpcontent/uploads/2009/09/LL-Uveitis-anterior-2011-11-30-Endversion.pdf (letzter Zugriff: 06.10.2017).

2 Heiligenhaus A, Michels H, Schumacher C, et al.: Interdisziplinäre Leitlinie zur Diagnostik und antientzündlichen Therapie der Uveitis bei juveniler ideopathischer Arthritis. www.dog.org/wp-content/uploads/2009/09/045012I_S2k_Uveitis_bei_juveniler_idiopathischer_Arthriti.pdf (letzter Zugriff: 06.10.2017).

3 Taylor HR, Keeffe JE: World blindness: a 21st century perspective. Br J Ophthalmol 2001;85:261-266.

4 Berufsverband der Augenärzte Deutschlands e.V.: Augenärzte informieren: Uveitis. cms.augeninfo.de/fileadmin/pat_brosch/uveitis.pdf (letzter Zugriff: 06.10.2017).

5 DocCheck Flexikon: Uveitis. flexikon.doccheck.com/de/Uveitis (letzter Zugriff: 06.10.2017).

6 Pleyer U, Pohlmann D, Stübiger N: Therapie der posterioren, nichtinfektiösen Uveitis: Aktueller Stand und künftige Entwicklungen. Ophthalmologe. 2016;113:380-390.

7 Berufsverband der Augenärzte Deutschlands e.V., Deutsche Ophthalmologische Gesellschaft e.V.: Leitlinie Nr. 24b: Nichtinfektiöse Uveitis posterior. http://augeninfo.de/leit/leit24b.pdf (letzter Zugriff: 11.10.2017).

Prof. Dr. Uwe Pleyer, FEBO, Klinik für Augenheilkunde, Charité - Universitätsmedizin Berlin, Campus Virchow-Klinikum 